\title{
Resonant photoemission in single-crystal black phosphorus
}

\author{
M. Taniguchi* \\ Synchrotron Radiation Laboratory, Institute for Solid State Physics, The University of Tokyo, Tanashi, Tokyo 188, Japan \\ J. Ghijsen ${ }^{\dagger}$ and R. L. Johnson \\ Max-Planck-Institut für Festkörperforschung, Heisenbergstrasse 1, D-7000 Stuttgart 80, Federal Republic of Germany \\ S. Suga \\ Synchrotron Radiation Laboratory, Institute for Solid State Physics, The University of Tokyo, Tanashi, Tokyo 188, Japan \\ Y. Akahama and S. Endo \\ Research Center for Extreme Materials, Osaka University, Toyonaka, Osaka 560, Japan
}

(Received 29 September 1988)

\begin{abstract}
Resonant photoemission in single-crystal black phosphorus has been reinvestigated in detail by high-resolution spectroscopy. Partial-yield, valence-band, and constant-initial-state spectra were measured in the $2 p$ core-exciton excitation region. We have confirmed two types of resonances at the $2 p$ core threshold: one is a strong core-exciton-induced resonance with two final valence holes and the other is a weak Fano-type resonance. The Fano-type resonance is preferentially associated with the lower-energy spin-orbit doublet $\left(A-A^{\prime}\right)$ of the core excitons ( $A-A^{\prime}$ and $B-B^{\prime}$ being separated by $0.2 \mathrm{eV})$. This result elucidates the difference of the degree of localization of the core excitons, $A-A^{\prime}$ and $B-B^{\prime}$, and rules out the possibility that the $L_{2,3} V V$ Auger tail is solely responsible for the resonance profile.
\end{abstract}

Features of core-level absorption spectra in semiconductors depend strongly on the nature of electron-corehole $(e-c h)$ interaction. When the $e$-ch interaction is very weak or negligible, the spectra reflect the density of states (DOS) of unoccupied conduction bands. ${ }^{1}$ On the other hand, the strong $e$-ch coupling leads to a pronounced excitonic enhancement of the absorption near the core absorption threshold. ${ }^{2,3,4}$ In addition, the decay processes of such core excitons influence significantly the photoemission cross section of valence bands. ${ }^{2,4}$

In the case of black phosphorus $(\mathrm{P})$, the $2 p$ core absorption spectra have shown intense and sharp spin-orbit doublets, $A-A^{\prime}$ and $B-B^{\prime}$, due to the core-exciton formation. ${ }^{3}$ Moreover, the photoemission intensities of valence bands remarkably change near the $2 p$ core threshold. The strong increase of the photoemission intensity in the region of the upper $3 s$ valence bands has been interpreted as due to core-exciton-induced resonant photoemission with two final valence holes, whereas the weak change of the topmost $3 p$ valence-band intensity has been ascribed to Fano-type resonance. ${ }^{5} \mathrm{~A}$ detailed analysis of the two types of resonances, however, was restricted by a limited resolution of the photoemission spectra.

From an experiment made with a better resolution, Takahashi et al. claimed an absence of both types of core-exciton-derived resonances in black P. ${ }^{6}$ On the other hand, Nakano and Kotani investigated theoretically the resonant photoemission in black $P$ in detail and revealed an important role of core excitons in both resonances ${ }^{7}$ from comparison of the calculated spectra with the experimental data. ${ }^{6}$ The resonant photoemission in black $\mathbf{P}$ has been thus quite controversial.
Under such circumstances, we report in this paper on higher-resolution experiments of the resonant photoemission in black $\mathbf{P}$ crystal for three different configurations of the electric vector of light $(\mathbf{E})$ with respect to crystal axes. The present high-resolution experiments have partly corrected the earlier spectrum for the weak Fano-type resonance measured with a poor resolution. ${ }^{5}$ Two types of resonances, however, are confirmed to be taking place at the $2 p$ core threshold, in contrast to the conclusion by Takahashi et al. ${ }^{6}$ The strong enhancement at the upper $3 s$ bands is explained again as due to the core-excitoninduced resonance in the form of a resonant $L_{2,3} V V$ Auger process with two final holes in the valence bands. ${ }^{5,7}$ The weak Fano-type resonance observed at the topmost $3 p$ valence bands is found to be mainly due to an interference with the direct recombination process of the $\boldsymbol{A}-\boldsymbol{A}^{\prime}$ core-exciton doublet. Among them, the coreexciton-induced resonant $L_{2,3} V V$ Auger decay constitutes the dominant decay process. The results for black $\mathbf{P}$ single crystal are compared with those for GeS, GeSe, $\mathrm{SnS}$, and SnSe single crystals with black $\mathrm{P}$ structure, which exhibit a pronounced Fano-type resonance due to core excitons at the cation $d(\mathrm{Ge} 3 d$ and $\mathrm{Sn} 4 d$ ) core thresholds. ${ }^{4}$ The quite different features between black $\mathbf{P}$ and GeS-SnSe single crystals are successfully interpreted on the basis of a model proposed for the decay mechanism of core excitons in GeS-SnSe. ${ }^{4}$

The present experiments were performed at the Flipper II beam line of HASYLAB (Ref. 8) by making use of synchrotron radiation from the DORIS storage ring in Hamburg. A combination of a high-resolution plane-grating monochromator and a double-pass cylindrical mirror 
electron-energy analyzer was used to measure angleintegrated photoemission spectra. The exit slit of the monochromator was set to $200 \mu \mathrm{m}$ (Ref. 8) in order to obtain a sufficient counting rate in the photoemission measurements. The core absorption spectra were obtained by measuring partial-yield spectra at a final electron kinetic energy of $4.0 \mathrm{eV}$ with a window of $0.3 \mathrm{eV}$. The constant-initial-state (CIS) spectra, which trace the photoemission cross section of selected regions of valence bands as a function of photon energy $(\hbar \omega)$, were recorded directly by a synchronous scanning of the window energy of the electron-energy analyzer and $\hbar \omega$. Samples were high-purity single crystals of black $P$, whose in situ cleaved $a-c$ plane was used for measurements. Their orientation $^{3}$ was checked by low-energy electron diffraction (LEED) and $x$-ray diffraction. The working pressure was below $2 \times 10^{-10}$ Torr.

Figure 1(a) shows the $2 p$ core absorption spectra for $\mathbf{E} \| \mathbf{a}$ measured by means of partial yield (upper curve) and total yield with very high resolution $(\sim 30 \mathrm{meV}$ at 100
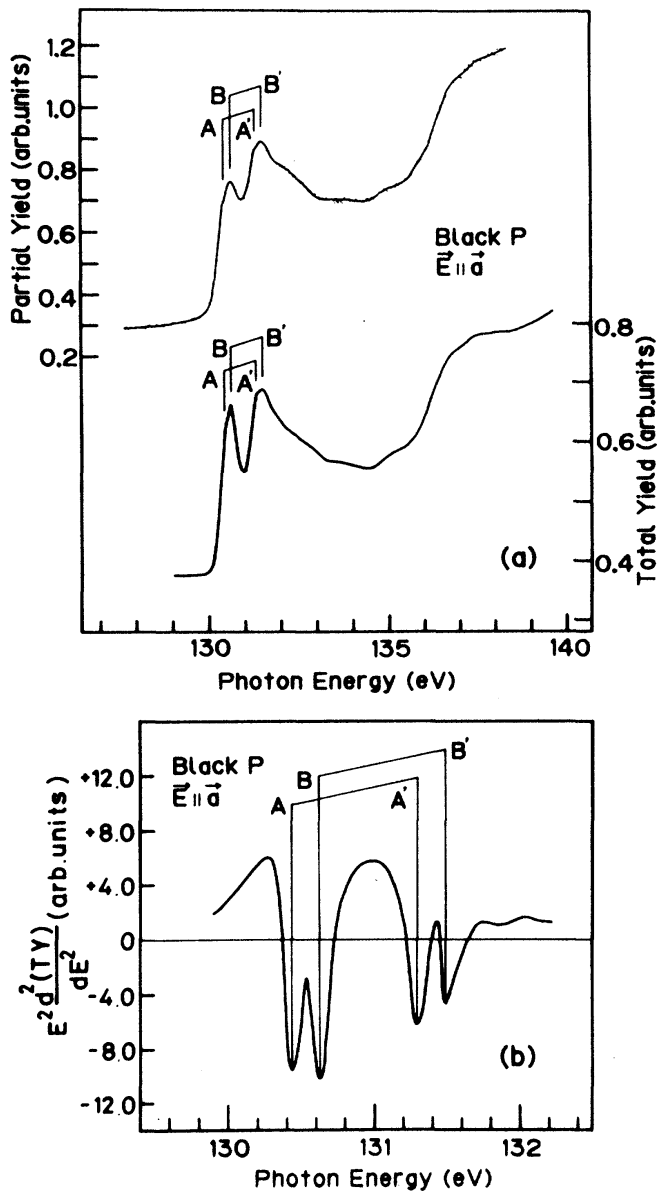

FIG. 1. (a) $2 p$ core absorption spectra of black $\mathbf{P}$ for $\mathbf{E} \| \mathbf{a}$ measured by means of partial yield (upper curve) and total yield with very high resolution (lower curve; from Ref. 3 ). The spinorbit doublets labeled $A-A^{\prime}$ and $B-B^{\prime}$ are due to the $2 p$ core excitons. (b) Second energy derivative of the total-yield spectrum (from Ref. 3 ). The shoulder structure $A$ is clearly resolved from the structure $B$.
$\mathrm{eV}$; lower curve). ${ }^{3}$ The second energy derivative of the total-yield spectrum is also shown in Fig. 1(b). The partial- and total-yield spectra agree well with each other by taking the different resolution into account. ${ }^{3,8}$ In addition, one notices that the $2 p$ core spectra show welldefined sharp peaks at the core absorption threshold separated by the spin-orbit-splitting energy of the initial states $(0.87 \mathrm{eV}) .^{3}$ The spin-orbit doublets $A-A^{\prime}$ and $B-B^{\prime}$ have been assigned to the $2 p$ core excitons associated with the quasi-two-dimensional conduction bands. ${ }^{3}$ The intrinsic full width at half maximum of the core-exciton absorption lines $A$ and $B$ has been estimated to be $0.3 \mathrm{eV}$ from a line-shape fitting.

Figure 2 shows a series of valence-band spectra of black $\mathbf{P}$ for $\mathbf{E} \| \mathbf{a}$ for $\hbar \omega$ between 127 and $134 \mathrm{eV}$. The intensities are normalized to the monochromator output and the binding energy $\left(E_{i}\right)$ is referred to the valenceband maximum. The valence-band spectra exhibit two groups of structures. A group extending from $E_{i}=0$ to 8 $\mathrm{eV}$ is predominantly derived from the $3 p$ states with a small contribution from the $3 s$ states, while another group of bands at $E_{i}=10.6$ and $\sim 15 \mathrm{eV}$ is mainly due to the $3 s$ states. One notices that the intensity of the peak at $E_{i}=10.6 \mathrm{eV}$ is remarkably enhanced without changing its peak position between $\sim 129$ and $130.6 \mathrm{eV}$. Above $\hbar \omega=132 \mathrm{eV}$, an additional peak shows up on the higherbinding-energy side of this peak. The new peak can be assigned to a standard $L_{2,3} V V$ Auger structure from its energy shift against $\hbar \omega$.

Details of the resonant behavior in Fig. 2 can be best studied in the form of CIS spectra. Such spectra for $\mathbf{E} \| \mathbf{a}$ are presented for selected valence bands at $E_{i}=2.6$ and $10.8 \mathrm{eV}$ in Fig. 3. The correlation of the CIS spectra with

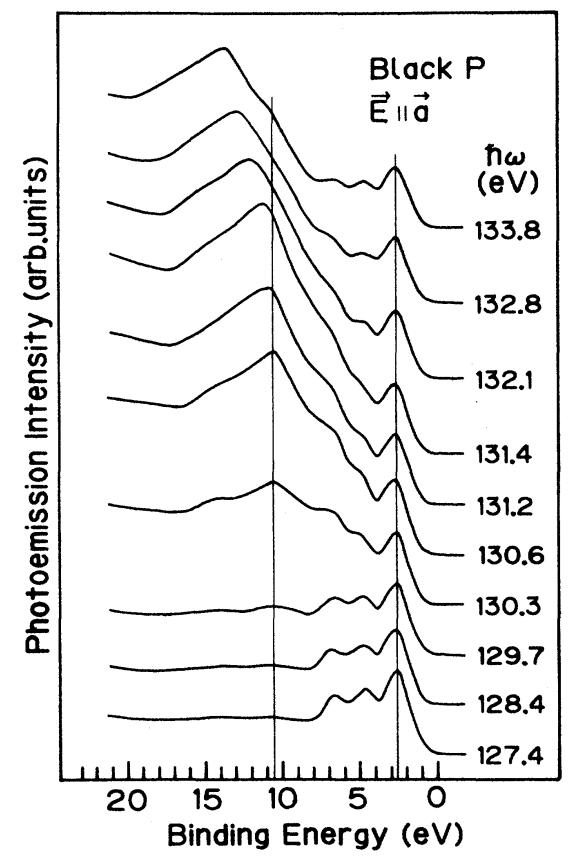

FIG. 2. A series of valence-band photoemission spectra of black $\mathbf{P}$ for $\mathbf{E} \| \mathbf{a}$ for $\hbar \omega$ between 127 and $134 \mathrm{eV}$. 


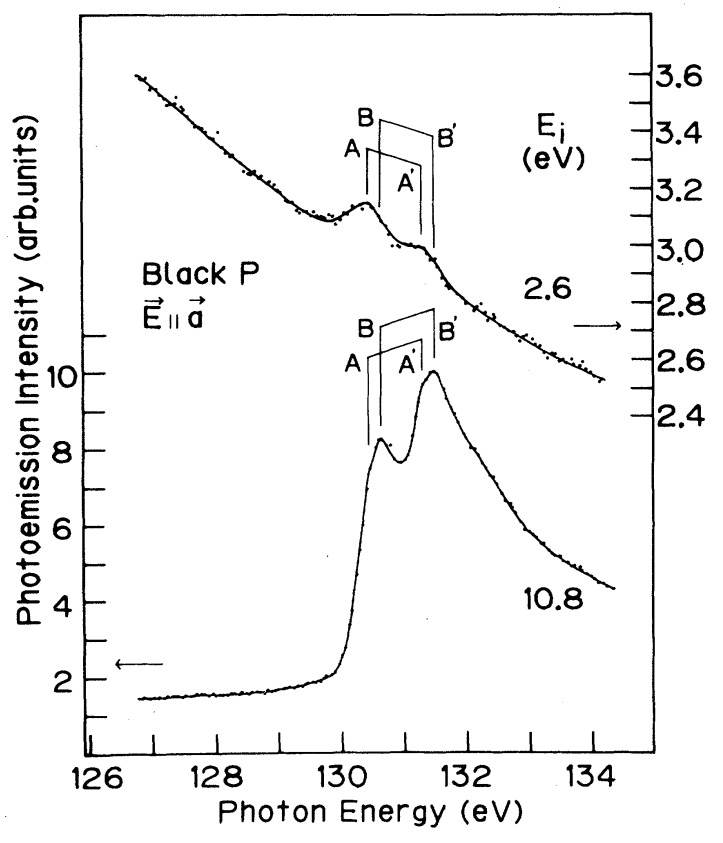

FIG. 3. CIS spectra of black $\mathbf{P}$ for $\mathbf{E} \| \mathbf{a}$ recorded at $E_{i}=2.6$ and $10.8 \mathrm{eV}$. Vertical lines $A-A^{\prime}$ and $B-B^{\prime}$ represent the energy positions of the $2 p$ core-exciton absorption.

the $2 p$ core-exciton excitation is apparent by a comparison with the corresponding absorption spectra in Fig. 1. The energies of the core-exciton absorption are marked by vertical lines, $A-A^{\prime}$ and $B-B^{\prime}$ in Fig. 3 , for the convenience of discussions below.

The CIS spectrum at $E_{i}=10.8 \mathrm{eV}$ exhibits intense and sharp peaks at the same energies as those of the $2 p$ core excitons in the absorption spectrum in Fig. 1. In addition, these peaks are followed by a tail in the higherenergy region. It is apparent that the intense and sharp doublets $A-A^{\prime}$ and $B-B^{\prime}$ are not due to the standard $L_{2,3} V V$ Auger transitions with a free conduction electron in the intermediate state, but are strongly subjected to the core-exciton effect, for the following reasons. The density of states (DOS) of conduction bands has no sharp peak structure around the lowest-energy region. This has been partly verified from the comparison of the calculated DOS (Ref. 9) with the $1 s$ and $2 s$ core absorption spectra, ${ }^{3}$ which reflect well the DOS of conduction bands. Therefore, if the $e$-ch interaction were weak or negligible, the intense and sharp doublets $A-A^{\prime}$ and $B-B^{\prime}$ would be observed neither in the CIS spectrum nor in the absorption spectrum. Instead, we would have only a broad structure in the CIS spectrum due to the standard $L_{2,3} V V$ Auger transition following the photoexcitation of a free conduction electron and a $2 p$ core hole, as pointed out by Nakano and Kotani ${ }^{7}$ for the extreme case of vanishing $e$ ch interaction. Such a standard $L_{2,3} V V$ Auger process contributes to the tail in the high-energy region of the CIS spectrum at $E_{i}=10.8 \mathrm{eV}$ in Fig. 3. In the case of the $2 p$ core absorption in black $\mathbf{P}$, however, the $e$-ch coupling is strong enough to produce sharp peaks in the absorption spectrum as shown in Fig. 1. Such a core-exciton effect necessarily appears in the intermediate state of the Auger decay process at the core excitation threshold. The Auger process under this circumstance is called the core-exciton-induced resonant Auger process. The $2 p$ core electrons are excited into the core-exciton bands with strong oscillator strengths at various $\hbar \omega$ within the bandwidth, dominated by the lifetime broadening due to the Auger decay of the core-hole state. The Auger filling of the core hole through the core-exciton $L_{2,3} V V$ Auger process results in a disappearance of the core exciton. Then, we find one kinetic electron and one conduction electron as well as two valence holes in the final state. This type of core-exciton-induced resonant process, which provides a corresponding photoemission structure at a constant binding energy, ${ }^{5,7}$ is a dominant source of the two sharp doublet structures in the CIS spectrum at $E_{i}=10.8 \mathrm{eV}$.

On the other hand, the CIS spectrum at $E_{i}=2.6 \mathrm{eV}$ shows only a weak doublet. Here, it should be noticed that the peak energies of the weak doublet coincide beyond the experimental uncertainty with those of the $A-A^{\prime}$ core-exciton doublet. In addition, the spectral shape is asymmetric with a weak dip on the low-energy side of the peak $A .{ }^{10}$ These features cannot be explained by the contribution of the $L_{2,3} V V$ Auger tail ${ }^{6}$ to the CIS structure because of the following reasons. First, if the tail contribution were significant, a second doublet would clearly appear at the position of the $B-B^{\prime}$ doublet. Even considering the statistics of the data points, no trace of the resonance structure is found in those energy regions. Second, the tail contribution is about 1 order of magnitude too weak to explain the experimental CIS structure. $^{7}$ The CIS spectrum, therefore, can be understood as due to a Fano-type resonance, which originates from an interference between the direct recombination process of the $2 p$ core excitons $\left(A-A^{\prime}\right)$ providing kinetic electrons from the $3 p$ valence bands and the direct excitation process of the $3 p$ valence electrons. The dominance of the $A-A^{\prime}$ doublet in the CIS spectrum reveals that the $B-B^{\prime}$ excitons have more extended character than the $A-A^{\prime}$ excitons, since the direct recombination process is favored by the localized character of the excitons.

Similar experiments were performed also for configurations of $\mathbf{E} \| \mathbf{c}$ and of $\mathbf{E}$ parallel to the $b-c$ crystal plane with an angle of $45^{\circ}$ between $\mathbf{E}$ and $\mathbf{c}$ (Ref. 6) $\left(E-45^{\circ}\right)$. These CIS spectra at $E_{i}=10.8 \mathrm{eV}$ exhibit two spin-orbit doublets, $A-A^{\prime}$ and $B-B^{\prime}$, at the same energies as those in the spectrum for $\mathbf{E} \| \mathbf{a}$. We found weak anisotropy for $\mathbf{E}\|\mathbf{a}, \mathbf{E}\| \mathbf{c}$, and $E-45^{\circ}$ configurations as regards the depth of the dip between the $B$ and $A^{\prime}$ peaks. The anisotropy is consistent with the results of the optical spectra. ${ }^{3}$ The CIS spectra at $E_{i}=2.6 \mathrm{eV}$, on the other hand, exhibit no conspicuous anisotropy for the abovementioned three configurations, except for the slightly different backgrounds. ${ }^{11}$ Regarding the splitting energy of the spin-orbit doublet in the CIS spectra at $E_{i}=2.6$ $\mathrm{eV}$, the present value of $0.87 \mathrm{eV}$ differs from that of 0.70 $\mathrm{eV}$, which can be estimated from the previous spectrum reported by Takahashi et al. ${ }^{6}$ Taking the statistics into account, the present CIS spectrum is considered to be more accurate than the previous one. ${ }^{6}$ 
It is worthwhile to compare the results for black $P$ with those for GeS, GeSe, SnS, and SnSe single crystals with black $\mathrm{P}$ structure. ${ }^{4}$ The cation core (Ge $3 d$ and $\mathrm{Sn}$ $4 d$ ) absorption spectra of GeS-SnSe show intense and sharp core-exciton doublets at the core absorption thresholds. The CIS spectra at the topmost $p$-valenceband peaks, which correspond to that at $E_{i}=2.6 \mathrm{eV}$ in black P, exhibit pronounced Fano-type resonances at the core absorption thresholds. The amount of the amplitude is nearly equal to or more than $100 \%$ of the background intensity. On the other hand, the $M_{4,5}\left(N_{4,5}\right) V V$ Auger emission is very weak. In GeS-SnSe, the states near the bottom of conduction bands are primarily derived from cation $p$ states, whereas the anion $p$ electrons contribute preferentially to the high-DOS features at the top of valence bands. Transitions from cation $d$ states to the states near the bottom of conduction bands are thus dipole-allowed intra-atomic transitions with strong oscillator strengths. The following decay of core excitons via the $M_{4,5}\left(N_{4,5}\right) V V$ Auger process has the character of an interatomic Auger decay due to the strong anion $p$ contribution to the top part of the valence band with high DOS. This accounts for the dominance of the direct recombination channel for the decay of the cationderived core excitons.

On the same line of argument, the $2 p$ core excitons in black $\mathbf{P}$ are excited by the dipole-allowed intra-atomic transitions with strong oscillator strengths. The following decay of the $2 p$ core hole via the core-exciton $L_{2,3} V V$ Auger decay is an intra-atomic type as well takes place with a fairly high probability, in contrast to the case of the cation $d$ core-derived excitons in GeS-SnSe. Accordingly, the contribution of the core-exciton $L_{2,3} V V$ Auger process is very strong in black $\mathbf{P}$ relative to that of the direct recombination process. This is the reason why the Fano-type resonance in black $P$ is much weaker than that in GeS-SnSe.

In conclusion, our high-resolution experiments have provided information on the resonant photoemission in black P, which could not be obtained from the experiments by Takahashi et al. ${ }^{6}$ due to their poor statistics and insufficient resolution. The new results and interpretation presented here should clarify and eliminate the existing controversy. We have confirmed (i) a strong coreexciton-induced resonance with two final valence holes, and (ii) a weak Fano-type resonance at the $2 p$ core threshold for three different configurations. The core-excitoninduced resonance (i) is observed as intense doublets ( $A$ $A^{\prime}$ and $\left.B-B^{\prime}\right)$ in the CIS spectrum at $E_{i}=10.8 \mathrm{eV}$. Occurrence of the Fano-type resonance (ii) is confirmed by the observation of a single doublet in the CIS spectrum at $E_{i}=2.6 \mathrm{eV}$, whose peak energies do not agree with those of the $B-B^{\prime}$ doublet but coincide with those of the $A-A^{\prime}$ doublet in the CIS spectrum at $E_{i}=10.8 \mathrm{eV}$. As concerns the decay of the $2 p$ core excitons, the contribution of the core-exciton $L_{2,3} V V$ Auger process is fairly strong relative to that of the direct recombination process, mainly due to an intraatomic character of the Auger process in black $P$.

We are grateful to Mr. H. Kölln for technical support, to Professor A. Kotani and Dr. T. Nakano for fruitful discussion, and to Professor M. Cardona for a critical reading of the manuscript. This project was partially financed by the Japan Society for the Promotion of Science, and by the German Minister for Research and Technology, under Grants No. 05-250-CA and No. 05390-CAB.
${ }^{*}$ Present address: Department of Materials Science, Faculty of Science, Hiroshima University, Higashisenda-machi, Nakaku, Hiroshima 730, Japan.

†Present address: Department of Applied Physics and Solid State Physics, University of Groningen, Nijenborgh 18, NL8747 AG Groningen, The Netherlands.

${ }^{1}$ W. Gudat, E. E. Koch, P. Y. Yu, M. Cardona, and C. M. Penchina, Phys. Status Solidi B 52, 505 (1972).

${ }^{2}$ G. J. Lapeyre and J. Anderson, Phys. Rev. Lett. 35, 117 (1975); F. Sette, P. Perfetti, F. Patella, C. Quaresima, C. Capasso, M. Capozi, and A. Savoia, Phys. Rev. B 28, 4882 (1983).

${ }^{3}$ M. Taniguchi, S. Suga, M. Seki, A. Mikuni, S. Asaoka, H. Kanzaki, Y. Akahama, S. Endo, and S. Narita, Phys. Rev. B 30, 4555 (1984).

${ }^{4}$ M. Taniguchi, J. Ghijsen, R. L. Johnson, and L. Ley, in Proceedings of the 18th International Conference on the Physics of Semiconductors, Stockholm, 1986, edited by O. Engström (World Scientific, Singapore, 1986), p. 1799.

${ }^{5}$ M. Taniguchi, S. Suga, M. Seki, H. Sakamoto, H. Kanzaki, Y. Akahama, S. Endo, S. Terada, and S. Narita, Solid State Commun. 49, 867 (1984).
${ }^{6}$ M. Takahashi, H. Ohsawa, N. Gunasekara, T. Kinoshita, H. Ishii, T. Sagawa, T. Miyahara, and H. Kato, Phys. Rev. B 33, 1485 (1986).

${ }^{7}$ T. Nakano and A. Kotani, J. Phys. Soc. Jpn. 55, 2867 (1986).

${ }^{8}$ R. L. Johnson and J. Reichardt, Nucl. Instrum. Methods 208, 791 (1983).

${ }^{9}$ H. Asahina, K. Shindo, and A. Morita, J. Phys. Soc. Jpn. 51, 1193 (1982).

${ }^{10}$ Intensity profiles of the 2.7 - and 10.8 -eV peaks in Ref. 5 have been found to be smoothly disordered due to a poor estimation of the intensity of the first-order light in the region overlapping with the tail of the zeroth-order light of the monochromator $(\hbar \omega>125 \mathrm{eV})$. The essential features are, however, qualitatively reproduced.

${ }^{11} \mathrm{CIS}$ spectrum at the topmost valence bands in Ref. 7 has been calculated by assuming Fano-type profiles with a negative $q$ factor at the energy positions corresponding to those of the $B-B^{\prime}$ core-exciton doublet, though the Fano-type resonance is found to be associated with the $A-A^{\prime}$ core excitons with a positive $q$ factor in the present experiments. This, however, does not require any essential change of the theory. 